\title{
La emergencia emotiva del pensamiento objetivo*
}

\author{
Camila Suárez Acevedo** \\ Miguel Ángel Pérez Jiménez
}

Recibido: 18 de febrero de 2018

Evaluado: 10 de abril de 2018

Aceptado: 7 de mayo de 2018

\section{Resumen}

El auge del externismo sobre los contenidos mentales ha motivado enfoques interpretativos sobre la atribución de pensamientos. La teoría de la triangulación destaca en ese contexto, pues hace compatibles el externismo físico y el social al señalar que la atribución de pensamientos responde a los estándares de racionalidad del intérprete aplicados a una base de evidencia constituida por la interacción causal y social del interpretado con su entorno. Esta dependencia externa causal y social del pensamiento es lo que lo hace objetivo. Sin embargo, la triangulación tiene problemas para explicar la atribución de pensamiento objetivo cuando la base evidencial es insuficiente, tal como ocurre en los primeros estadios del desarrollo psicológico. Este caso pone en evidencia un cúmulo de suposiciones no explicadas del externismo sobre las relaciones mente-mundo e interpretado-intérprete. El problema se debe a un error fundamental en la definición de los escenarios triangulares de interpretación, que puede subsanarse introduciendo algunas tesis sobre las emociones básicas que a su vez conllevan una redefinición del concepto mismo de triangulación.

Palabras clave: externismo, triangulación, emoción, Donald Davidson

\footnotetext{
Artículo de investigación. Resultado del proyecto "Aspectos éticos de la teoría cognitiva de la emoción de Robert Solomon" (ID PRY 005465) de la Vicerrectoría de Investigación de la Pontificia Universidad Javeriana de Bogotá. Citar como: Suárez, C y Pérez, M. (2018). La emergencia emotiva del pensamiento objetivo. Hallazgos, 15(30), 141-156. DOI: https://doi.org/10.15332/2422409X.4807

** Estudiante del Doctorado en Filosofía de la Pontificia Universidad Javeriana. Magistra en Filosofía de la misma universidad y Psicóloga egresada de la Universidad Santo Tomás. Contacto: guiovana.suarez@javeriana.edu.co.

*** Doctor en Filosofía por la Universidad de Granada y profesor asociado del Departamento de Filosofía de la Pontificia Universidad Javeriana de Bogotá. Licenciado y Magíster en Filosofía de la Pontificia Universidad Javeriana. Realizó Estudios avanzados (DEA) en Lógica y filosofía del lenguaje en la Universidad de Granada. Contacto: miguel.perez@javeriana.edu.co.
} 


\title{
The emotive emergence of objective thought
}

\begin{abstract}
Davidson's theory of triangulation is a brilliant exponent of externalists theories of mental content. According to triangulation, thoughts are attributed to an agent regarding evidential bases made of causal and social interactions. Thought is said objective in this precise sense, in that it depends both on world and other people to be ascribed. This kind of externalism must face the problem of objective thought attribution when evidential bases are insufficient, as when we must interpret nonverbal children. This difficulty raises because of a distorted conceptualization of triangulation scenarios. We propose a redefinition of triangulation that employs different sources of emotion theory. Our new conceptualization accounts for the objectivity of thought in cases of interpretation of verbal and nonverbal humans.
\end{abstract} Received: february 18, 2018 Evaluated: april 10, 2018 Accepted: may 7, 2018

Keywords: externalism, triangulation, emotion, Donald Davidson. 


\section{A emergência emotiva do pensamento objetivo}

Recebido: 18 de fevereiro de 2017

Avaliado: 10 de abril de 2018 Aceito: 7 de maio de 2018

\section{RESUMO}

O auge do externismo sobre os conteúdos mentais tem motivado enfoques interpretativos sobre a atribuição de pensamentos. A teoria da triangulação destaca nesse contexto, ora faz compatíveis o externismo físico e o social ao assinalar que a atribuição de pensamentos responde aos padrões de racionalidade do interprete aplicados a uma base de evidencia construída pela interação causal e social do interpretado com seu entorno. Esta dependência externa causal e social do pensamento é que o faz objetivo. Porem, a triangulação tem problemas para explicar a atribuição de pensamento objetivo quando a base evidencial é insuficiente, tal como acontece nos primeiros estados do desenvolvimento psicológico. Em este caso se evidencia um cúmulo de suposições não explicadas do externismo sobre as relações mente-mundo e interpretado-interprete. O problema é causado por um erro fundamental na definição dos cenários triangulares de interpretação, que se pode corrigir introduzindo algumas teses sobre as emoções básicas que a sua vez acarretam uma redefinição do conceito mesmo de triangulação.

Palavras-chave: externismo, triangulação, emoção, Donald Davidson. 


\section{INTRODUCCIÓN}

El auge de las posiciones externistas sobre los contenidos mentales ha motivado enfoques interpretativos sobre la atribución de pensamientos, de los que la teoría de la triangulación es el exponente más destacado, al menos en tanto hace compatibles los distintos tipos más reconocidos de externismo: el físico y el social (Davidson, 2001). Para la triangulación, la atribución recta de pensamientos responde a dos criterios: 1) la estructura holista del pensamiento; y 2) los estándares de racionalidad del intérprete aplicados a una base de evidencia constituida por la interacción causal y social del interpretado con su entorno. En este texto se analizará el segundo de esos requisitos:1 la base evidencial que soporta la objetividad del pensamiento. A este respecto se debe anotar que la triangulación se ve en problemas para explicar la atribución de actitudes cuando la base evidencial es insuficiente, tal como ocurre en los estadios de la aparición del pensamiento. Considerar este caso pone en evidencia un cúmulo de suposiciones no explicadas sobre las relaciones mundo-pensamiento e interpretado-intérprete en este tipo de teorías. El problema de la explicación externista se debe a un error fundamental en la definición de los escenarios triangulares de interpretación, que puede subsanarse introduciendo algunas tesis sobre las emociones básicas que a su vez conllevan una redefinición del concepto mismo de triangulación.

El primero es el más ampliamente discutido en la literatura. Para más información se puede ver en Pérez 2011, 2015, 2016a y 2016b.

\section{EL EXTERNISMO}

INTERPRETATIVO Y LA OBJETIVIDAD DEL PENSAMIENTO

Aun cuando el último medio siglo ha visto un creciente auge de teorías externistas sobre el contenido mental, no puede decirse que exista en el presente una definición precisa y comúnmente aceptada de lo que es. Dos ejemplos de ello son Rudd, quien considera el externismo "como la doctrina de que la mente no se contiene a sí misma, de que para comprender nuestros estados mentales es esencial referirnos a hechos del entorno social o físico en los que estamos situados" (Rudd, 1997, p. 501). Por su parte, Davidson sostiene que el externismo es "una manera de ver según la cual la conexión entre el pensamiento y el mundo es algo intrínseco más que extrínseco -es una conexión no inferida, ni construida, ni descubierta, sino que está allí desde el comienzo" (Davidson, 2001, p. 2).

Estas definiciones tienen puntos de contacto y de divergencia que ilustran dos rasgos esenciales de toda posición externista: su referencia a un mundo externo y a una relación entre la mente y ese mundo externo. En cuanto a las diferencias es destacable que para Rudd el externismo tiene relación con nuestra comprensión de los estados mentales y no con su naturaleza. Para Davidson en cambio es claro que la relación entre el pensamiento y el mundo es constitutiva, los estados mentales son por naturaleza externistas. Estas diferencias se dan respecto a la manera de concebir qué relación hay entre el mundo y el pensamiento. Sin embargo, las dos definiciones coinciden en que siempre hay una referencia del pensamiento al mundo externo. 
La idea de mundo externo que se juega en el externismo ha sido fuente de polémica, y ha motivado una distinción entre externismo social (Burge, 1979; Kripke, 1982) y externismo físico (Putnam, 1975; Burge, 1988). Para el primero, son los hechos del mundo social o las prácticas de las comunidades las que guardan una relación estrecha con los estados mentales. Para el segundo, son los hechos del mundo natural y las interacciones causales los que afectan al pensamiento. ${ }^{2}$

Cualquiera de estas dos variedades de externismo puede a su vez subdividirse especificando cómo entienden la relación que hay entre los hechos del mundo externo y los pensamientos. Podrán establecerse relaciones más o menos fuertes que motivan tipos diferentes de externismo, entre los que se destacan las siguientes: en primer lugar, un externismo metafísico según el cual el pensamiento tiene relaciones constitutivas con el mundo externo por principio, forma parte esencial de la naturaleza del pensamiento tener estas relaciones constitutivas con el mundo externo. En segundo lugar, un externismo genético según el cual los pensamientos tienen su origen en el mundo externo. En tercer lugar, un externismo interpretativo para el que la atribución e identificación de pensamientos, según nuestra forma corriente de hablar, está sujeta a las condiciones del

2 Burge ha notado que hay una estrategia argumentativa común para los dos tipos de externismo que consiste en "mantener constante la historia del movimiento corporal de la persona, de los estímulos en su superficie y de su química interna. Luego, mediante una variación del entorno con el que la persona interactúa, mientras se mantienen constantes los efectos moleculares sobre el cuerpo de la persona, uno puede mostrar que algunos de los pensamientos de sus pensamientos varían" (Burge, 1988, p. 650). mundo externo. En las líneas que siguen se estudiará el externismo interpretativo. ${ }^{3}$

Las teorías interpretativas referidas suelen entender los pensamientos, en la tradición de Frege, como actitudes con un contenido proposicional definido (Frege, 1918). En este sentido, las creencias son los tipos de pensamiento por excelencia ya que cualquier otra actitud depende de ellas. No es posible tener deseos, intenciones, anhelos o esperanzas sin albergar algunas creencias. Por eso, la creencia es la actitud básica (Davidson, 1975). Dichas teorías, además, suelen reconocer varios rasgos a este tipo de actitudes, como su objetividad, su carecer holista y cuando se las examina semánticamente exhiben intensionalidad (Davidson, 1997a).

Desde un punto de vista externista, el rasgo más llamativo del pensamiento es su objetividad. Al respecto es necesario precisar que "nuestras creencias son objetivas no por estar libres de prejuicios y por haberse formado a la luz de toda la evidencia, por supuesto, sino en el sentido de que son verdaderas o falsas, y de que, con pocas excepciones, su verdad depende de cuestiones que no dependen de nosotros" (Davidson, 2001, p. 1). Es esta objetividad la que ancla el pensamiento fuera de la mente. Por eso el primer reto para una teoría externista general del pensamiento es explicar su objetividad. Sin embargo, en el contexto específico del externismo interpretativo aparece un reto adicional al constatar que sería poco plausible explicar cómo se le atribuyen pensamientos a una criatura, con base en evidencia externa,

3 Sobre hasta qué punto una posición externista interpretativa guarda compromisos realistas respecto a los pensamientos o a los contenidos mentales en general puede verse el debate en Davidson (1997b) y Dennett (1991). 
asumiendo que esa criatura ya tiene pensamientos. Un externismo interpretativo no sólo debe dar una explicación de cómo se atribuye pensamiento objetivo, sino que debe hacerlo sin presuponer que el sujeto sobre el que recae la atribución ya tiene pensamiento.

\section{EL EXTERNismo}

\section{INTERPRETATIVO Y LA TRIANGULACIÓN}

La teoría de la interpretación radical sobresale como una buena alternativa dentro del externismo interpretativo, pues se rige por la cuestión de cómo atribuir pensamientos a una criatura sin dar por sentado que dicha criatura tiene ya pensamientos (Davidson, 1973). El primer aporte de la teoría al externismo es diferenciar dos elementos que el intérprete debe tener en cuenta: 1) descifrar qué evidencia tiene de los supuestos pensamientos de una criatura; y 2) cómo puede emplear esa evidencia para realizar las atribuciones (Edwards, 1994).

Respecto del primer elemento, la interpretación radical sostiene que, puesto que debe tratarse de evidencia no psicológica, los recursos que tiene el intérprete son, por una parte, el comportamiento del agente a interpretar; y, por otra, el entorno en el que los dos se encuentran. Davidson solía ilustrar esta dependencia contextual del pensamiento respecto del hablante y de la situación mediante el concepto de la verdad. Un pensamiento es verdadero en virtud de los conceptos que lo componen, en relación con el interpretado y con el entorno (Davidson, 1974). Puesto que el sentido del externismo es, precisamente, anclar el pensamiento a su contexto, podemos decir que el primer elemento de la interpretación radical compromete a la teoría con un externismo físico representado por el concepto de la verdad.

El segundo elemento impone una nueva dificultad. Para fijar el pensamiento a atribuir es preciso poner en relación el comportamiento del interpretado con su entorno, es decir, establecer un vínculo entre los dos recursos de evidencia con que cuenta el intérprete. El problema es que esto se puede hacer de muchas maneras, el comportamiento no es unívoco al respecto. La manera como se establezcan las relaciones interpretado-contexto está en manos del intérprete, y ello lo fuerza a colocar algo de su parte. Por eso, a modo de principio propio, el intérprete atribuye pensamiento buscando preservar las características mismas del pensamiento. Las atribuciones tratan de hacer al interpretado, por una parte, un agente coherente; y, por otra, hasta donde sea posible, acertado en sus creencias respecto al entorno, de acuerdo con los estándares de coherencia y de corrección del propio intérprete (Davidson, 1991). Así entonces, la atribución de pensamientos asume que los pensamientos no son internos, ya no sólo respecto al entorno causal natural, sino también respecto de otros seres pensantes. Se asume que el pensamiento se comparte con una comunidad de pensadores y que guarda una relación estrecha con ella. Por esta razón el segundo elemento compromete a la teoría también con un externismo social.

Esta caracterización del empleo de los recursos mundanos como evidencia en la tarea interpretativa hace patente que las dos variedades de externismo consideradas inicialmente, el físico y el social, resultan insuficientes para satisfacer el externismo 
interpretativo. El primer elemento obliga a que sean los hechos del mundo natural los que fijen el contenido, pero por las exigencias del segundo la única manera de poder aprovechar los hechos como evidencia es recurrir a una atribución favorable de racionalidad al hablante, según los estándares del intérprete, esto introduce un externismo social a la atribución. Por estas razones es preciso introducir un externismo de doble aspecto, un externismo triangular, que sitúe el pensamiento en relación con dos tipos de coordenadas: el mundo natural y el mundo social (Davidson, 2001). Sólo este tipo de externismo parece adecuado para la recta atribución de pensamientos. ${ }^{4}$

El externismo triangular no es sólo una síntesis del externismo causal y el social. Es más bien una comprensión triangular de las mutuas dependencias que hay entre la subjetividad, la intersubjetividad y la objetividad. En este sentido es un externismo metafísico. Sin embargo, en lo que sigue, se establecerá cómo se dan las relaciones de apoyo evidencial entre esos tres factores a la hora de atribuir pensamientos, de modo que el análisis se mantendrá en el terreno interpretativo. ${ }^{5}$ El interés es poner a prue-

4 Hemos atado el externismo causal y el externismo social a dos elementos distintos de la interpretación radical, a saber: el concepto de verdad y el principio de caridad, respectivamente. Esto es sólo una estrategia discursiva, pues tanto la convención V como el principio de caridad ponen en evidencia los dos tipos de externismo. La primera, pues en ella se explicita la relación formal que hay entre las lenguas del interpretado y del intérprete; el segundo, pues, a través del principio caritativo de correspondencia, el intérprete puede asumir la conducta del hablante situada en un entorno causal presuntamente compartido como evidencia para verificar o refutar sus atribuciones de pensamiento. En este sentido, nuestra tesis es que la interpretación radical se acoge a un externismo triangular, tanto en los aspectos formales como en los de verificación mediante evidencia empírica.

5 La palabra "evidencia" resulta ambigua en este contexto. Por una parte, puede significar la relación de apoyo evidencial que hay entre dos ba la adecuación del externismo triangular examinando cómo enfrenta los problemas de insuficiencia de evidencia para la atribución de pensamientos, tal como ocurre en la aparición del pensamiento. ${ }^{6}$

\section{EL EXTERNISMO TRIANGULAR Y LA APARICIÓN DEL PENSAMIENTO}

El problema de la aparición del pensamiento se puede plantear desde diversos enfoques. En el contexto del externismo interpretativo la cuestión es por qué se le atribuye pensamiento a una criatura cuando antes no se hizo, si sólo se hacen dichas atribuciones con base en evidencia externa. El problema parece estructurarse en dos momentos: primero no existe evidencia suficiente para atribuir pensamiento, pero luego sí la hay. Aquí aparecen dos alternativas: o bien el cambio en la evidencia es lo que motiva que se empiece a atribuir pensamientos, o bien es que se aplica el principio de caridad para suplir las insuficiencias que deja la evidencia. Por ahora se analizará la primera alternativa. El problema es que, en primer lugar, se tiene una evidencia no psicológica que no justifica atribuciones psicológicas sino sólo explicaciones causales. Luego, en segundo

pensamientos, por otro la relación que hay entre el comportamiento contextual de un agente y un intérprete que se sirve de dicho comportamiento para atribuir pensamiento al agente. En el primer caso la evidencia debe ser de carácter proposicional y la llamaremos justificación. Reservaremos el término 'evidencia' para usarlo en su segundo sentido. Exposiciones de las relaciones de dependencia que forjan el externismo metafísico triangular son Davidson (1991 y 2001).

6 La estrategia más común para poner a prueba el externismo, en especial sus versiones epistemológicas, es decir, las que consideran que la justificación del conocimiento se basa en evidencias externas, es el debate del conocimiento de uno mismo, pues en este caso no parece necesaria evidencia externa alguna para establecer la verdad o la certeza de los conocimientos que uno tiene de sí mismo (Ludlow y Martin, 1998). 
lugar, se tiene una evidencia no psicológica que sí justifica atribución psicológica. ¿Qué sucedió en el tránsito?

Una manera de enfrentar la cuestión es examinar los criterios de evidencia existentes para utilizar explicaciones causales o explicaciones psicológicas. Como se veía desde la interpretación radical, la evidencia base de la explicación de un agente, sea esta causal o psicológica, es su comportamiento en un entorno. La diferencia en las explicaciones reposa en que, cuando el comportamiento del agente es poco sofisticado, la explicación causal basta. Sin embargo, puesto que hay comportamientos demasiado sofisticados como para ser agotados por la explicación causal, la explicación psicológica es la que mejor se les adecua (Davidson, 1997a; Dennett, 1971). Desde un punto de vista interpretativo también hay una diferencia semántica entre los dos tipos de evidencia externa que motivan o no las atribuciones de pensamiento. Cuando la evidencia es insuficiente, es posible describirla causalmente con vocabulario extensional. Cuando la evidencia es suficiente, se puede describir psicológicamente utilizando vocabulario intencional.

Estas observaciones sobre el tipo de explicación que puede emplearse para la aparición del pensamiento conducen a una conclusión pesimista que adopta la forma de una contradicción. Cuando una criatura está en los estadios intermedios su comportamiento no es del todo simple, pero tampoco es lo suficientemente complejo. Así, puede que deba ser interpretada como causal y no causal, o como racional y no racional, al mismo tiempo. Sin embargo, puesto que el vocabulario es o bien causal o bien psicológico, nada de lo que se diga en estos casos es satisfactorio, no hay consistencia en ninguno de los dos marcos explicativos: aunque lo parezca, un bebé no es un termostato que reaccione causalmente; pero un bebé tampoco es un agente psicológico con creencias, deseos e intenciones, aunque lo parezca.

Consecuente con esta conclusión, Davidson vio siempre problemática la posibilidad de una psicología evolutiva. Sin embargo, sostuvo que era posible describir las condiciones necesarias y suficientes del pensamiento. Las dos condiciones describen una situación "que involucra dos o más criaturas simultáneamente en interacción unas con otras y con el mundo que comparten, es lo que llamo triangulación" (Davidson, 1997a, p. 183). La condición necesaria es esta interacción primitiva que se da incluso entre criaturas no racionales, como un banco de peces, por ejemplo. La condición suficiente es ese mismo tipo de interacción modificada cualitativamente de modo que todas las criaturas que intervengan en ella sean racionales, agentes capaces de comunicarse lingüísticamente entre sí. Por eso Davidson suele decir que las condiciones del pensamiento son una interacción social primitiva contextualizada; y la comunicación, condiciones en las que se hace explícito el externismo de doble aspecto causal y social de la triangulación (Davidson, 1997a).

El problema de la analogía de la triangulación como explicación de la aparición del pensamiento es que hereda de la interpretación radical la brecha semántica entre las explicaciones causales y las psicológicas (Glüer, 2006; Miguens, 2006). La primera condición del pensamiento es completamente causal y la segunda completamente psicológica, por eso no se ponen en contacto 
en ningún momento dejando por fuera la posibilidad de dar una explicación de la aparición del pensamiento. Esta problemática conclusión fuerza a plantearse otras cuestiones. Puesto que la evidencia de la explicación psicológica es el comportamiento contextualizado sofisticado, es decir, el comportamiento lingüístico, la pregunta es ¿cómo se puede reconocer? A pesar de la simplicidad de la pregunta, las triangulaciones no pueden responderla. En la triangulación preconceptual no hay criaturas lingüísticas, luego, no hay lenguaje qué reconocer. En la triangulación conceptual sí hay lenguaje, pero sólo hay lenguaje, de modo que allí no hay evidencia no psicológica para realizar la atribución no circular de pensamientos. Por eso la triangulación davidsoniana es tanto insuficiente como circular para explicar la aparición del pensamiento y, por tanto, es inadecuada como una teoría interpretativa externista.

Sin embargo, a pesar de lo problemática que es la triangulación, su introducción permite un avance respecto a la teoría de la interpretación radical: introduce la posibilidad de considerar agentes completamente causales en la interacción interpretativa, y eso no estaba en dicha teoría donde todo agente era de entrada cognitivo y lingüístico. El problema es que estos recursos introducidos no se han explotado suficientemente bien. En adelante se seguirá un camino alternativo en el uso de la analogía de la triangulación. Para hacerlo se introducirá el concepto de triangulación transicional. Se trata de diseñar un escenario interpretativo donde la interacción triangular se da entre una criatura conceptual, una criatura no conceptual y un entorno compartido según el criterio de al menos alguna de las criaturas. Esta descripción de la triangulación transicional cobija varios escenarios posibles, como la interacción de una criatura racional con un animal, o con algunos aparatos. Sin embargo, se limitará el escenario a la interacción entre un agente racional y un bebé.

La triangulación transicional difiere de los escenarios clásicos de interpretación radical y también de la triangulación clásica. En esos escenarios, el intérprete toma tanto el comportamiento lingüístico del hablante como las condiciones del entorno como evidencia para la atribución de pensamientos. En la triangulación transicional, por el contrario, el intérprete no tiene evidencia comportamental lingüística para soportar sus atribuciones de pensamiento. Los bebés no hablan, así que el intérprete carece de evidencia, no tiene herramientas conceptuales apropiadas para relacionar el comportamiento del bebé con rasgos específicos del entorno. El comportamiento del bebé no es lo suficientemente complejo como para que el intérprete le pueda atribuir actitudes proposicionales definidas. A pesar de esto, el intérprete podría apelar a la caridad para compensar la falta de evidencia. El problema es que, careciendo de la posibilidad de atribuir actitudes con contenido, el intérprete pierde el criterio para evaluar la coherencia, la correspondencia y la racionalidad en general, de los estados mentales que atribuya al bebé. Como consecuencia, el intérprete tampoco puede considerar los comportamientos del bebé como acciones propiamente dichas, no los puede racionalizar, y entonces ni siquiera puede considerar su razonabilidad según los estándares del principio de caridad. ${ }^{7}$

7 En realidad, podría obtenerse una conclusión positiva indicando que los intérpretes son capaces de asumir una actitud caritativa no 
Sin embargo, aunque Davidson está en lo cierto al negar que se tiene buena evidencia para atribuir pensamiento a los bebés o a los animales, no es menos cierto que por todas partes se encuentran personas tratando a los bebés, e incluso a algunos animales, como agentes racionales. Más llamativo todavía resulta que este injustificado trato racional unilateral parece tener una importante influencia en el buen desarrollo de los seres humanos y de muchos animales también. Si se deja de lado el pesimismo de creer que en este caso se está frente a una atribución arbitraria de pensamientos, es válido preguntar de qué evidencia se valen los intérpretes para realizar esas impropias pero apropiadas atribuciones a criaturas preconceptuales en escenarios de triangulación transicional. De un modo general la pregunta es ¿cuáles son las condiciones de la interpretación psicológica preconceptual?

\section{EMOCIONES BÁSICAS Y EXTERNISMO TRIANGULAR}

Davidson mostró que hay dos condiciones para que el pensamiento aparezca: una interacción social primitiva y la comunicación que permite la objetividad del pensamiento. El primer problema es que no se ha especificado cómo se establecen los lazos iniciales entre las criaturas en la interacción primitiva, simplemente se los ha dado por supuestos. El segundo problema es que tampoco se ha dado ninguna indicación de cómo puede dicha interacción sofisticarse hasta permitir la objetividad. Por el contrario, se ha definido la comunicación como una condición

proposicional en estos casos. En nuestra opinión esta es una opción correcta que motiva la introducción de un principio emotivo de caridad. Hemos dado algunos pasos en esta dirección introduciendo la idea de una segunda persona emotiva (Pérez, 2008). por completo diferente a la interacción social primitiva. En lo que sigue se mostrará que si se considera a los agentes de la triangulación transicional como agentes emotivos estos dos problemas pueden recibir una respuesta satisfactoria. ${ }^{8}$

La intersubjetividad del externismo triangular supone una particular comprensión de la mente que se aparta de quienes la identifican con el comportamiento, pues en esta perspectiva carece de sentido atribuir pensamientos; y de quienes los divorcian, pues de entrada destierran la posibilidad de tener dos agentes que puedan establecer alguna relación entre sus pensamientos por vía de sus comportamientos.

Distintos teóricos de la psicología y la filosofía han mostrado que una de las condiciones de la interacción comunicativa no conceptual es una dimensión puramente práctica no verbal de la misma, y que incluso la comunicación verbal descansa en un cimiento amplio, sólido pero falible, de interacciones no verbales (Eilan, 2005; Glock, 1996; Wittgenstein, 1953). La interacción comunicativa presupone capacidades para reconocer gestos, muecas, expresiones faciales o diversas entonaciones vocales, por destacar sólo fenómenos de nivel personal. En realidad, esto es una verdad de Perogrullo, sin embargo, no suele dársele el lugar que merece en las explicaciones de la interacción, sin duda

8 No ofreceremos una explicación detallada de ninguno de los dos aspectos. En especial no entramos a exponer el desarrollo completo de la objetividad desde su emergencia hasta su estabilización en el concepto de la verdad. Intuiciones parecidas a la nuestra sobre las raíces emotivas del pensamiento y sobre el papel de las emociones en la triangulación pueden encontrarse en Moya (1994), Pedace (2017) y Quintanilla (2004). La primera de ellas se mueve a un nivel más básico que el nuestro, el de las sensaciones, mientras que la segunda y la tercera exploran las posibilidades alternativas que abre los conceptos de simpatía y empatía. 
debido a la hegemonía de las teorías proposicionales de la acción y de la comunicación, como las del propio Davidson. A la sombra de las palabras, los gestos parecen mudos, en la triangulación transicional, por el contrario, la elocuencia del gesto cobra protagonismo. ${ }^{9}$ Lo decisivo de estos aportes es haber mostrado que el componente gestual no es intrínseco al comportamiento, sino un tipo de calificación suya, una modalidad de los comportamientos que permite la interacción (Pickard, 2003). La comprensión preconceptual de lo mental no se juega entonces al nivel del comportamiento sino de esas cualidades del comportamiento que, genéricamente hablando, son los gestos.

La variación cualitativa del comportamiento por excelencia es la emotividad, y en realidad uno de los rasgos más destacados de la emotividad es que centra la atención de quien la presencia en ella más que en el propio comportamiento o que en el contenido proposicional (Ben-Ze'Ev, 2003). Por ejemplo, cuando el novio pide disculpas a la novia, mientras conserva una expresión despreocupada al hablar, o como cuando el niño dice a su madre, con voz temblorosa, que no ha roto el jarrón mientras suda y su rostro está enrojecido.

En la teoría de las emociones básicas estas modalidades de la conducta se explican por la existencia de "programas de afecto", que son dispositivos biológicos adaptativos que nos hacen sudar, cambiar la entonación vocal e incluso desequilibran el balance

9 Davidson jamás permaneció ajeno por entero a la necesidad de estas capacidades de expresión y percepción gestual que están a la base de cualquier interacción posible entre una criatura y su entorno natural o social (Davidson, 2001 y Glüer, 2006). Sin embargo, se limitó a mencionarlas y suponerlas sin llegar a tratar de darles algún contenido. hormonal en una situación específica (Ekman, 1999, p. 46). Las emociones básicas se caracterizan, aunque no exclusivamente, por un componente dual judicativo y expresivo. El primer componente involucra una forma de percepción automática que discrimina dando a la situación percibida cierto valor como favorable o desfavorable para quien la percibe. La valencia de la emoción se debe a su origen evolutivo destinado a la supervivencia. El entorno primitivo favorece o no y las emociones cualifican la percepción presentando un entorno valorativamente cargado en este sentido (Ekman, 1999). El segundo componente conlleva una serie de manifestaciones de la emoción que constituyen propiamente la modalidad del comportamiento. Muchas de estas manifestaciones, las más básicas, son universales, pero también pueden acomodar reacciones culturalmente aprendidas. Estas manifestaciones, que son tanto de nivel personal como subpersonal, son especialmente fáciles de reconocer y entre ellas se ha notado una especial relevancia de la expresión facial como manifestación del estado emocional (Ekman, 1999).

Desde el punto de vista de la intersubjetividad, estos dos aspectos hacen de las emociones básicas candidatos idóneos para ocupar un lugar destacado en la triangulación transicional. ${ }^{10}$ En primer lugar, gracias

10 Es común tratar a Davidson como un autor que favorece una teoría cognitiva de las emociones, como la de Robert Solomon, por ejemplo (Green, 2013). Esta idea haría incompatible a Davidson con una teoría de las emociones básicas. Nuestra propuesta en este texto tampoco es decir que Davidson podría acogerse a la teoría de las emociones básicas, sino sólo que hay rasgos no cognitivos de las emociones que las hacen insumos de notable valor para la interacción no conceptual. Las relaciones de las ideas de Davidson con las distintas teorías de las emociones son complejas y no es fácil encasillarlo en el cognitivismo, ni en el naturalismo y ni siquiera en las teorías suigeneristas (Pérez, 2015). 
a su carácter dual los programas de afecto son capacidades tanto para cualificar emotivamente los propios comportamientos como para reconocer los rasgos emocionales del comportamiento de otros. Por eso la hipótesis de los programas de afecto es sugerente para comprender cómo comienza la intersubjetividad (Griffiths, 2003 y Pickard, 2003). En segundo lugar, gracias a que funcionan como modalidades de la acción, las emociones guardan cierta independencia de los contenidos proposicionales que se atribuyen para interpretar las acciones, aunque son compatibles con ellos (Griffiths, 2003). Tras este breve comentario sobre el aporte de las emociones a la explicación del surgimiento de la intersubjetividad y con ella del externismo social; para terminar, se estudiará su posible contribución al surgimiento de la objetividad.

¿Pueden las emociones dar una explicación de cómo llega un niño a tener la idea de mundo objetivo?, ¿en qué sentido y hasta qué punto el intercambio emocional puede dotar a una criatura con la idea de una realidad independiente de ella y del sujeto con el que interactúa? La idea davidsoniana de objetividad como "independencia de la mente" parece ser intuitivamente clara, pero hay un sentido en que no lo es. El problema radica en que se puede relacionar rápidamente la "independencia" del objeto respecto a la mente con la idea de un tercer elemento en la interacción, algo que no es el niño ni el que lo cuida. Pero esto no es obvio, “ ¿dónde comienza o termina un objeto?, ¿es el propio pie un 'objeto' separado de uno mismo?, ¿es el movimiento del propio pie un objeto?, ¿son objetos nuestros rostros o nuestras sonrisas?, ¿es la interacción misma un objeto?, cuándo tomas la mano de alguien, ¿es una de las manos un objeto?" (Reddy, 2005, p. 88).

Los teóricos de la atención conjunta han mostrado que los primeros estadios de desarrollo se dan en relaciones diádicas, sin "tercer elemento" por decirlo así (Hobson, 2005, p. 188). El tercer elemento aparece tardíamente cuando el niño se hace consciente de que está vinculado en las relaciones que el otro mantiene con un mundo independiente. Para llegar a esta conciencia superior el niño ha debido ser involucrado en las actividades del que lo cuida y sólo así se reconoce como parte de esa interacción. ${ }^{11}$ Esto sugiere que la idea de un tercer elemento no responde exactamente a la idea de interacción triádica. Precisamente, lo que sucede es que hay un tercer elemento en la interacción diádica: la primera experiencia de objeto del bebé es que él mismo es objeto. En la interacción diádica el bebé descubre las respuestas emocionales del que lo cuida como respuestas a un objeto externo al propio cuidador (Reddy, 2005). ¿Qué puede ser ese objeto si no él mismo? La intuición básica de esta propuesta es que el sentido de mundo objetivo del niño comienza cuando él empieza a saber de sí en relación con su cuidador. ${ }^{12}$ Con

11 Esta es la explicación de por qué es preciso introducir un principio emotivo de caridad. Debemos tratar al bebé como si fuera ya un agente racional de modo que mantengamos interacciones normativas con él aunque no tengamos evidencia ni autorización externa para hacerlo. Esta idea es decisiva también más adelante en el proceso de desarrollo cuando el niño está aprendiendo a usar el lenguaje. Todavía no habla bien, pero hay que tratarlo como si pudiera hacerlo para poder corregirlo y que entonces efectivamente llegue a hacerlo (Franco, 2005).

12 El trabajo sobre niños con autismo ha mostrado que su desconexión del mundo objetivo es el resultado de una deficiencia emocional. Nunca llegaron a tener un mundo compartido porque jamás lograron establecer relaciones intersubjetivas como las que hemos descrito, interacción emocional. Ahora bien, si el primer objeto de la triangulación es el propio niño, la conclusión es que el niño jamás logra reconocerse como otro para alguien. Según la hipótesis de la interacción diádica y 
Davidson, la interacción social es la fuente de la objetividad, pero el surgimiento de la objetividad puede no tener relación con un "tercer elemento" fuera de los agentes, sino entre los agentes (Reddy, 2005).

Esta importante precisión ayuda a entender en qué sentido el intercambio emocional es la fuente primaria de la objetividad. Sin embargo, el sentido de un mundo objetivo no se agota en saberse objeto para otro, pues involucra la idea de una realidad independiente de las mentes en general. Sin una independencia tal, la intersubjetividad no sería la fuente de la objetividad sino la objetividad misma. Es preciso entonces dar alguna explicación de cómo se expande la comprensión del yo como objeto al resto del mundo, por decirlo así.

La sugerencia de Reddy es que las interacciones con el otro son sólo una de las fuentes en la expansión de la idea de objetividad (Reddy, 2005). Antes de que aparezca el "tercer elemento" en el sentido de Davidson, hay una relación que se establece entre el bebé y sus propias acciones. Dentro de ellas se destaca una que parece dirigirse hacia la idea de una realidad independiente del propio niño: el señalamiento declarativo. Curiosamente éste es el gesto más característico de los bebés frente a los primates y a niños con autismo (Gómez, 2004; Franco, 2005). ${ }^{13}$

Según las teorías experienciales de la atención conjunta, el señalamiento protodeclarativo

el tercer elemento, el autismo sería fruto de una deficiencia emocional que se reporta no sólo en la falta de un sentido de mundo objetivo sino también en la falta un sentido de sí mismo (Hobson, 2005).

13 Un estudio completo de las variedades del gesto de señalar y de los trabajos experimentales más recientes sobre el particular es el de Franco (2005). Nosotros no consideraremos todas las variaciones que ella contempla; en todo caso sus conclusiones son útiles y muy compatibles con las ideas que aquí exponemos. es la manifestación de una posición contemplativa del niño frente a un mundo independiente de él. A diferencia de los gestos protoimperativos, comunes a varios tipos de animales, que se caracterizan por ser una forma de interacción instrumental con el entorno, los protodeclarativos parecen mostrar un tipo de relación desinteresada del bebé con el mundo. Sin duda el entorno les es llamativo, pero, a diferencia de los primates, que algo sea llamativo no es sólo una cuestión de supervivencia, al menos para el bebé en el segundo año de vida. Esto se ha afirmado al comprobar que el bebé se muestra satisfecho cuando el adulto simplemente presta atención a lo que él le indica, aunque no se lo alcance o haga alguna otra acción con el objeto.

Sin embargo, lo que más ha llamado la atención del gesto protodeclarativo es su estrecho parecido con la función lingüística de la referencia (Franco, 2005). Si se permite la expresión, las condiciones de satisfacción de un acto de habla declarativo y las de un señalamiento protodeclarativo son las mismas: que el gesto se ajuste al mundo. La diferencia esencial entre ambos estaría en que, en el primer caso, la corrección del ajuste estaría dada por el concepto de verdad; pero en el segundo caso, no se tiene una noción preconceptual equivalente, exceptuando quizá la equívoca referencia a la "correspondencia con la cosa".

La propuesta de Franco se apoya en que los bebés no sólo manifiestan agrado o desagrado por las personas sino también por los objetos (Franco, 2005). Cuando el niño señala al objeto puede sonreír o llorar, lo mismo que cuando se le aproxima el objeto o cuando se lo aleja. Estas manifestaciones 
emocionales expresan un parecer subjetivo del niño respecto al objeto que Franco llama un "comentario sobre el mundo" y que califica como "predicaciones primitivas".

Así pues, las interacciones emocionales vinculan no sólo al niño con el adulto en la intersubjetividad, sino también al niño con los objetos en la predicación primitiva. Esta alternativa permite comprender que las emociones juegan un rol decisivo en la aparición del pensamiento y serían entonces el elemento de transición básico entre los dos tipos de triangulación de Davidson. Las emociones son la columna vertebral de la triangulación transicional, pues garantizan la interacción social primitiva y el surgimiento de la objetividad. Por eso el externismo triangular, en la medida que conjuga los aspectos sociales y causales de la triangulación no debe caracterizarse como cognitivo, o como no cognitivo sino como un externismo triangular emotivo.

\section{REFERENCIAS}

Ben-Ze'Ev, A. (2003). The Logic of Emotions. En A. Hatzimoysis (Ed.), Philosophy and the Emotions (pp. 147-162). Cambridge, EUA: Cambridge University Press.

Burge, T. (1979). El individualismo y lo mental. En L. M. Valdés(Comp.), Pensamiento y Lenguaje. Ciudad de México, México: UNAM.

Burge, T. (1988). Individualism and Self-Knowledge. Journal of Philosophy, 85, 649-663.

Davidson, D. (1973). Interpretación radical. En G. Filipi(Trad.), De la verdad y de la interpretación. Barcelona, España: Gedisa.

Davidson, D. (1974). La creencia y el fundamento del significado. En G. Filipi
(Trad.), De la verdad y de la interpretación. Barcelona, España: Gedisa.

Davidson, D. (1975). Pensamiento y habla. En G. Filipi(Trad.), De la verdad y de la interpretación. Barcelona, España: Gedisa.

Davidson, D. (1991). Tres variedades de conocimiento. En O. Fernández (Trad.), Subjetivo, intersubjetivo, objetivo (pp. 280300). Madrid, España: Cátedra.

Davidson, D. (1997a). La aparición del pensamiento. En O. Fernández (Trad.), Subjetivo, intersubjetivo, objetivo (pp. 176190). Madrid, España: Cátedra.

Davidson, D. (1997b). El indeterminismo y el antirrealismo. En O. Fernández (Trad.), Subjetivo, intersubjetivo, objetivo (pp. 110-129). Madrid, España: Cátedra.

Davidson, D. (2001). Externalisms. En P. Kotatko, P. Paginy G. Segal (Eds.), Interpreting Davidson. Stanford, EUA: CSLI Publications.

Dennett, D. (1971). Intentional Systems. The Journal of Philosophy, 68(4), 87-106. doi:10.2307/2025382

Dennett, D. (1991). Brainchildren. Essays on Designing Minds. Londres, Inglaterra: The MIT Press.

Edwards, S. (1994). Externalism in the Philosophy of Mind. Aldesdorf, Alemania: Averbury.

Eilan, N. (2005). Joint Attention Comunication and Mind. En N. Eilan, et al. (Eds.), Joint Attention: Communication and Other Minds (pp. 1-33). Oxford, Inglaterra: Oxford University Press.

Ekman, P. (1999). Basic Emotions. En T. Dalgleish y M. Power (Eds.), Handbook of Cognition and Emotion (pp. 45-60). Nueva York, EUA: John Wiley \& Sons.

Franco, F. (2005). Infant Pointing: Harlequin, Servant of Two Masters. En N. Eilan, et al. (Eds.). Joint Attention: Communication 
and Other Minds (pp. 129-164). Oxford, Inglaterra: Oxford University Press.

Frege, G. (1918). El pensamiento. Una investigación lógica. En L. M. Valdés (Trad.), Investigaciones lógicas. Madrid, España: Tecnos.

Glock, H. J. (1996). On Safari with Wittgenstein, Quine and Davidson. En R. Arrington y H. J. Glock (Eds.), Wittgenstein and Quine (pp. 144-172). Londres, Inglaterra: Routledge.

Glüer, K. (2006). Triangulation. En E. Lepore y B. Smith (Eds.), The Oxford Handbook of Philosophy of Language. Oxford, Inglaterra: Oxford University Press.

Gómez, J. C. (2004). El desarrollo de la mente en los simios, los monos y los niños. Madrid, España: Morata.

Green, M. (2013). The Rationality of Emotions. En E. Lepore y K. Ludwig (Eds.), A Companion to Donald Davidson (pp. 506-518). Oxford, Inglaterra: John Wiley \& Sons.

Griffiths, P. (2003). Basic Emotions, Complex Emotions, Machiavelian Emotions. En A. Hatzimoysis (Ed.), Philosophy and the Emotions (pp. 36-68). Cambridge, Inglaterra: Cambridge University Press.

Hobson, P. (2005). What Puts the Jointness into Joint Attention. En N. Eilan et al. (Eds.), Joint Attention: Communication and Other Minds (pp. 185-204). Oxford, Inglaterra: Oxford University Press.

Kripke, S. (1982). Wittgenstein on Rules and Private Language. Oxford, Inglaterra: Oxford University Press.

Ludlow, P. y Martin, N. (1998). Externalism and Self-Knowledge. Stanford, EUA: CSLI Publications.

Miguens, S. (2006). Conceito de crença, trianulações e atencão conjunta. En
Perspectives on Rationality. Porto: Faculdade de Letras da Universidade do Porto.

Moya, C. (1994). Las emociones y la naturalización de la intencionalidad. Anales del seminario de metafísica, 28, 227-255.

Pedace, K. (2017). Mente y lenguaje. La filosofía de Donald Davidson, modelo para armar. Buenos Aires, Argentina: SADAF

Pérez, M. A. (2008). First, Second and Third Person in the Emergence of Thought. En J. Padilla-Galvez (Ed.). Phenomenology as Grammar (pp. 185-197). Frankfurt, Germany: Ontos Verlag.

Pérez, M. A. (2011). Un primate de tercera y una persona de segunda. Sobre el valor del rostro la mirada y la piel para comprender a un extraño. Universitas Philosophica, 28 (57), 265-293.

Pérez, M. A. (2015). De Davidson a la teoría de la emoción, y vuelta. Co-herencia, 23, 141-170.

Pérez, M. A. (2016a). Racionalidad animal. Un debate psicológico en perspectiva hermenéutica. En Forero, J. A. (Ed.). Racionalidad, lenguaje y acción. Aproximaciones analíticas (pp.35-58). Bogotá, Colombia: Editorial de la Universidad del Minuto de Dios.

Pérez, M. A. (2016b). La ontología tras bastidores. Un problema metafísico para el naturalismo genético. Universitas Philosophica, 33 (66), 61-76.

Pickard, H. (2003). Emotions and the Problem of Other Minds. En A. Hatzimoysis (Ed.), Philosophy and the Emotions (pp. 87-104). Cambridge, Inglaterra: Cambridge University Press.

Putnam, H. (1975). El significado de "significado". En J. J. Acero (Trad.). Teorema, 14, 345-405. 
Quintanilla, P. (2004). Comprender al otro es crear un espacio compartido. Ideas y Valores, 125, 117-133.

Reddy, V. (2005). Before the 'Third Element': Understanding Attention to self. En N. Eilan et al. (Eds.). Joint Attention:
Communication and Other Minds (pp. 85109). Oxford, Inglaterra: Oxford University Press.

Rudd, A. (1997). Two Types of Externalism. The Philosophical Quarterly, 47 (189), 501-507.

Wittgenstein, L. (1953). Investigaciones filosóficas. En García, A. y Moulines, U. (Trad.). Ciudad de México, México: UNAM-Crítica. 